\title{
Serum Alanine Aminotransferase Elevations in HIV Positive Patients on Antiretroviral Therapy in Namibia
}

\author{
Lovis P. Shanyengana, Munyaradzi Mukesi*, Berta E. van der Colf, Sylvester R. Moyo \\ Department of Health Sciences, Faculty of Health and Applied Sciences, Namibia University of Science and Technology, Windhoek, Namibia \\ Email: ^mmukesi@nust.na
}

How to cite this paper: Shanyengana, L.P., Mukesi, M., van der Colf, B.E. and Moyo, S.R. (2016) Serum Alanine Aminotransferase Elevations in HIV Positive Patients on Antiretroviral Therapy in Namibia. World Journal of AIDS, 6, 101-110. http://dx.doi.org/10.4236/wja.2016.63014

Received: July 14, 2016

Accepted: September 25, 2016

Published: September 28, 2016

Copyright $\odot 2016$ by authors and Scientific Research Publishing Inc. This work is licensed under the Creative Commons Attribution International License (CC BY 4.0).

http://creativecommons.org/licenses/by/4.0/

\begin{abstract}
Elevated alanine aminotransferase (ALT) levels in Human Immunodeficiency Virus (HIV) infected people is a major concern in the world and especially in Africa. It may lead to liver failure and even death. Certain antiretroviral (ARV) drugs, such as nevirapine and efavirenz, are known to cause toxicity. Other causes of elevated ALT are viral hepatitis, the HIV virus itself and other drugs such as anti-tu- berculosis drugs and alcoholism. The study aimed at determining the prevalence of elevated ALT levels in HIV positive patients on antiretroviral therapy during the period 2013 to 2014. This was a retrospective study which included 267 patient records from Katutura and Windhoek Central hospitals in Windhoek, Namibia. The subjects' ages ranged from 21 to 82 years. The patients enrolled were on the first line treatment and their ALT levels were recorded at each monitoring period. ALT levels, viral hepatitis results and the antiretroviral therapy (ART) regimen were the most important aspects included in the study. Out of 267 patients, $18 \%$ had ALT elevation associated with grade 1 to 4 toxicity levels. The study found that $1.4 \%$ of patients developed severe liver toxicity (grade 3 and 4 toxicity). Toxicity occurred throughout the treatment period but was the highest at six months of treatment. Patients on nevirapine based regimens had lower toxicity compared to those receiving efavirenz based regimens. Patients who had HIV and viral hepatitis co-infection had high toxicity although the study found no severe hepatotoxicity in these patients.
\end{abstract}

\section{Keywords}

ARV, Toxicity, ALT, Namibia, Nevirapine, Efavirenz

\section{Introduction}

In 2008, an estimated 22.4 million people were living with HIV in sub-Saharan Africa 
(SSA) [1]. Based on 2008 antenatal sero-surveillance data, an estimated $17.8 \% \mathrm{Na}$ mibians aged 15 - 49 years were infected with HIV [2]. Namibia had an estimated 14.3\% HIV prevalence between the ages of 15 and 49 years in 2013. Windhoek was estimated to have 30,000 people living with HIV/AIDS with an HIV prevalence for the age group 15 - 49 years of $15.8 \%$ in 2011 [3]. In 2015, the prevalence rate declined to $13.6 \%$ [4]. Namibia is among the countries with high HIV prevalence rates in the world. According to the Global HIV/AIDS-Namibia statistics (2015), the reduction of HIV related deaths and improvement in the quality of people living with HIV (PLHIV) are the main goals for the treatment, care and support program [4]. The large scale rollout of ART has resulted in a significant decline in HIV-related morbidity and mortality [1] [5].

Since the introduction of antiretroviral drugs, Human Immunodeficiency Virus (HIV) infection management has become increasingly complex. HIV positive patients are receiving an array of drugs to treat and prevent opportunistic infection. With the increased availability of antiretroviral therapy (ART), more people are now surviving with HIV but more are presenting with increasing liver disease [6]. This has consequently caused an increase in hepatic complications. In Namibia, 105,347 adults were reported to be receiving antiretroviral therapy (ART) in 2012 with a projected $91 \%$ ART coverage [7].

Certain antiretroviral (ARVs) drugs have well known documented toxicity. Although almost all drugs can cause toxicity, those found to cause liver toxicity include nevirapine, efavirenz, abacavir and lamivudine [6] [8]. Liver disease is emerging as an important co-morbidity in HIV-infected patients in low and middle income countries and Namibia is no exception. According to the previous studies conducted in other African settings, elevated ALT levels were estimated to be approximately 13\% in HIV patients on ART [9].

Clinical studies have indicated that grade 3 hepatotoxicity was observed in approximately 5\% - 10\% of HIV-positive patients treated with combination ART for 16 months. A retrospective cohort study that determined the incidence of NNRTI hepatotoxicity in a group of HIV-infected patients in New York City practice found that grade 3 - 4 elevations in ALT and/or AST levels occurred in 3 (1.1\%) of 272 patients [10]. Another study in the US concluded that severe hepatotoxicity occurred throughout the course of NNRTI-based therapy and was more common among patients prescribed with NVP (occurring in 15.6\%) than those prescribed with efavirenz (8.0\%) [11] [12]. However, it is likely that co-infection with viral hepatitis was a contributing factor [12]. In a Switzerland cohort of 2365 HIV-infected individuals not co-infected with either HBV or HCV, 385 (16\%) had chronically elevated ALT (defined as $>2 \times$ upper limit of normal) [6]. A post-mortem study of $86 \mathrm{HIV}$-infected individuals undergoing autopsy in rural South Africa demonstrated that $10 \%$ had liver related conditions at the time of death [13].

According to the Namibia National Guidelines on ART (2014), it is important to understand the therapeutic effects, side effects and challenges regarding the implemen- 
tation of highly active anti-retroviral therapy (HAART). HAART, which uses combinations of three ARV drugs in most cases, has proven to be far better than monotherapy or dual nucleoside regimens, which are not recommended due to rapid emergence of resistance and treatment failure. The recommended minimum laboratory tests before initiating ART are an HIV antibody test, haemoglobin or haematocrit level, a white blood cell count and differential and serum alanine or aspartate aminotransferase levels. Serum creatinine and urea are used to assess baseline renal function. A pregnancy test for women and serum glucose are also performed. Consequently, patients receiving ART are required to be monitored routinely. Routine tests include haematological and biochemical tests for the detection of drug toxicity and response to therapy. Treatment monitoring should have the capacity to recognise and appropriately manage common HIV related illnesses, opportunistic infections (e.g. Tuberculosis (TB) and adverse reactions to anti-retroviral (ARV) drugs [14].

Liver toxicity particularly is one of the many side effects of ART. Certain factors have been associated with the development of liver toxicity such as hepatitis co-infection, numerous other liver diseases, including alcohol-related injuries, drug-induced hepatotoxicity, metabolic fatty liver, vascular and autoimmune diseases, infectious diseases and hepato-biliary malignancies. These conditions can affect the clinical management and prognosis of HIV infection [1] [5] [8]. The severity of liver toxicity ranges from transient elevations in transaminases to hepatic failure and death [6]. ALT should be monitored subsequently at two weeks, six weeks, twelve weeks, six months and every six months there-after. Severe hepatotoxicity is defined as ALT levels more than five times the upper limit of the reference range (Grade 3 and 4). Grade 1 and 2 are considered as mild elevations and do not require discontinuation of the drug causing the elevations. Drug toxicity is graded according to serum ALT results as follows: Grade 0 (< $1.25 \times$ Upper Limit of Norma 1 [ULN]); Grade 1 (1.25 - 2.5× ULN); Grade 2 (2.6 - 5x ULN); Grade 3 (5.1 - 10× ULN) and Grade 4 (>10× ULN) [15].

Two Nucleoside Reverse Transcriptase Inhibitors (NRTI) and one Non Nucleoside Reverse Transcriptase Inhibitor (NNRTI) are used as first line drugs. Nevirapine is contraindicated in patients with higher CD4 cell counts $\left(>400\right.$ cells $/ \mathrm{mm}^{3}$ in men, $>250$ cells $/ \mathrm{mm}^{3}$ in women). However, this seems to be less important in well-controlled HIV infection [8]. Efavirenz (EFV) and nevirapine (NVP) were included in first line regimens for HAART although the first-line regimen for HAART in the public sector wasstavudine, lamivudine and nevirapine ( $\mathrm{d} 4 \mathrm{~T} / 3 \mathrm{TC} / \mathrm{NVP})$. The advantage of nevirapine is its possible use in pregnancy and its lower cost as a generic product. The advantage of efavirenz is its possible use in combination with TB treatment. First line drugs for tuberculosis, which is a common coinfection of HIV, such as rifampicin, isoniazid and pyrazinamide are also well known to cause liver toxicity. Rifampicin causes drug interactions with ART resulting in direct hepatotoxicity while isoniazid's hepatotoxicity increases in HIV infection and pyrazinamide results in dose-related toxicity [6].

There has been no published research on the prevalence of elevated liver enzymes in HIV positive patients in Namibia. Therefore the current study aimed at determining 
the prevalence of elevated ALT levels in HIV positive patients on ART in Windhoek.

\section{Research Study Design}

This was a retrospective study in which a total of 267 patient records were used, of which 144 records were from Windhoek Central hospital and 123 records from Katutura State hospital. None probability, judgmental sampling method was used to select the patient records. Baseline ALT was recorded and then two weeks, six weeks, three months, six months and one year after initiation of treatment. Creatinine and CD4 results were recorded at baseline and six months after treatment initiation. Hepatitis B surface antigen screening test was recorded only at baseline.

\section{Inclusion Exclusion Criteria}

HIV-infected patients who initiated ART treatment during the period 2013-2014 were included in the study. Adult HIV patients who had at least two months of follow up and those who started on first line ART during the study period were eligible for the study. Patients who did not have the ALT baseline results done or available were excluded. Patients who had treatment discontinued before the second follow up due to lack of treatment adherence and resistance were also excluded. In addition, patients who were transferred from other facilities were also excluded from the study. Before initiation of treatment patients were counselled on adherence, concomitant use of other drugs and alcohol intake during ART.

\section{Methods of Analysis}

The patient results used in the study were tested at the Namibia Institute Pathology (NIP). ALT, creatinine, HIV and hepatitis B virus surface antigen tests were analysed using the Architect analyser (Abbort, Illinois, USA). CD4 count was analysed using the Beckman Coulter Flow Cytometer 500/CellMek which uses the fluorescent flow-cytometry method (Beckman, Indianapolis, USA).

Data was analysed using the Statistical Package for Social Sciences (SPSS) version 22.0. The Pearson Chi-square $p$ value was used to determine whether a statistically significant association existed between variables. Using a 95\% confidence level, a $\mathrm{P}$ value of less than or equal to 0.05 ( $\mathrm{P} \leq 0.05)$, was considered to be statistically significant. The paired t-test was used to compare the monitoring periods ALT means to the initial ALT and determine whether the difference was significant or not. The independent t-test was used to compare the initial ALT mean and the one year ALT mean of the patients with viral hepatitis and those without viral hepatitis.

\section{Ethical Considerations}

Permission was granted by NUST and Ministry of Health and Social Services (MOHSS) research committees. Individual patient consent was not required as archived records were used and patient identification was concealed. 


\section{Results}

Of the 267 patients, 171 were females and 96 were males with ages ranging from $21-82$ years. These were patients visiting the ART clinic for routine monitoring. Routine counselling was done to ensure treatment adherence and reduce use and abuse of alcohol during the treatment period. This study did not assess the effects of alcohol on ALT. On average, patients started ARV treatment on a CD4 count of 262 cells $/ \mu \mathrm{L}$ and creatinine levels of $72 \mu \mathrm{mol} / \mathrm{L}$.

Patients were enrolled in two different first line treatment regimes, Tenofovir/ Lamivudine/Efavirenz (TDF/3TC/EFV), Tenofovir/Lamivudine/Nevirapine (TDF/3TC/ NVP). Ninety six patients were enrolled on TDF/3TC/EFV of which 30 were males and 66 were female and 159 were enrolled on TDF/3TC/NVP of which 59 were males and 100 were females.

The baseline ALT levels were used as the control group which indicated the levels of ALT in HIV positive patients who were not on treatment. A total of 267 patient records were used in the control group of which 96 were males and 171 were females. Patients who initiated treatment while they had elevated ALT levels were 45 (16.9\%) of which 25 were males and 20 were females. Of those patients who initiated treatment with elevated ALT, two patients (4.4\%) had unknown hepatitis status, nine patients (20\%) had viral hepatitis and $34(75.6 \%)$ did not have viral hepatitis. The mean ALT was 28.1 IU/L. ALT results were recorded two weeks after initiation of treatment, and subsequently after six weeks, three months, six months and one year. Table 1 shows mean ALT values at the different monitoring intervals.

Mean ALT level after initiation of treatment with the peak elevation observed at six months (49.4 IU/L). There was no significant difference in ALT mean between the baseline ALT and two weeks after treatment initiation ( $\mathrm{P}$ value: 0.082 ). The difference in mean ALT was statistically significant for the rest of the monitoring period when compared to the baseline ALT.

The treatment regimens were grouped into efavirenz based regimens and nevirapine based regimens. Table 1 shows different toxicity levels in the treatment regimens at the different monitoring periods.

Toxicity occurred throughout the treatment period. Nevirapine based treatment regime had toxicity that was increasing with the increase in treatment duration while those on efavirenz based treatment regime had varying toxicity throughout the monitoring period. The highest total toxicity of $26.5 \%$ (35 patients) and $28.2 \%$ (18 patients) in patients who were enrolled on nevirapine and efavirenz respectively were observed six months after initiation of treatment. Table 2 shows the total toxicity ratings in the different treatment regimens over the entire one year follow up period.

Total toxicity was not different between the two regimens. Mild toxicity was slightly higher in patients enrolled on nevirapine based treatment regimens (17.1\%) than those who were enrolled on efavirenz based treatment regimens (16.2\%). In contrast, severe toxicity was higher $(1.6 \%)$ in patients enrolled on efavirenz based treatment regimens than patients enrolled on nevirapine based treatment regimens (1.1\%). 
Table 1. Toxicity in the treatment regimens and monitoring periods.

\begin{tabular}{|c|c|c|c|c|c|c|c|}
\hline Monitoring intervals & ART regimen & (n) & $\begin{array}{c}\text { Grade } 1 \\
(\%)\end{array}$ & $\begin{array}{c}\text { Grade } 2 \\
(\%)\end{array}$ & $\begin{array}{c}\text { Grade } 3 \\
(\%)\end{array}$ & $\begin{array}{c}\text { Grade4 } \\
(\%)\end{array}$ & $\begin{array}{c}\text { Total Toxicity } \\
(\%)\end{array}$ \\
\hline Two Weeks & NVP-based & 164 & 10.2 & 0.4 & 0.0 & 0.0 & 10.6 \\
\hline Six Weeks & NVP-based & 75 & 8.0 & 4.0 & 1.3 & 1.3 & 14.6 \\
\hline Three months & EFV-based & 54 & 13.0 & 3.7 & 0.0 & 0.0 & 16.7 \\
\hline \multirow{2}{*}{ Six Months } & NVP-based & 132 & 21.9 & 4.5 & 0.0 & 0.0 & 26.5 \\
\hline & EFV-based & 64 & 21.9 & 4.7 & 0.0 & 1.6 & 28.2 \\
\hline
\end{tabular}

NVP-Nevirapine; EFV—Efavirenz.

Table 2. Total toxicity in treatment regimens.

\begin{tabular}{cccccc}
\hline & \multicolumn{2}{c}{ Mild toxicity } & \multicolumn{2}{c}{ Severe toxicity } \\
\hline Type of regime & Grade 1 (\%) & Grade 2 (\%) & Grade 3 (\%) & Grade 4 (\%) & Total (\%) \\
\hline NVP-based & 13.4 & 3.7 & 0.8 & 1.1 & 18.2 \\
Total & & 17.1 & & 0.5 & 1.1 \\
EFV-based & 13.8 & & 2.4 & & 1.6 \\
Total & & 16.2 & & & 17.8 \\
\hline
\end{tabular}

P value-0.99.

Out of 255 patients, 236 patients had hepatitis B surface antigen screening done while 19 did not have hepatitis screening test results. Out of the 236 patients who had viral hepatitis B screening test performed, 22 (9.3\%) tested positive for hepatitis B surface antigen. Table 3 shows toxicity levels at different monitoring periods for patients who initiated treatment with hepatitis $\mathrm{B}$ infection when compared to those who initiated treatment with no hepatitis B co infection.

Patients with viral hepatitis B had highest toxicity at two weeks (30.7\%) and three months (25\%) after initiation of treatment compared to those without viral hepatitis. Thereafter, toxicity was lower in those with hepatitis B virus in comparison to those without hepatitis B virus. However these toxicities were mild (grade 1 toxicity levels).

\section{Discussion}

The main objective of this study was to determine ALT levels in HIV positive patients on ART.

Mean ALT levels were higher during the monitoring period of two weeks, six weeks, three months, six months and a year after initiation of treatment compared to the base 
Table 3. Toxicity in patients on ART with and without viral hepatitis co-infection.

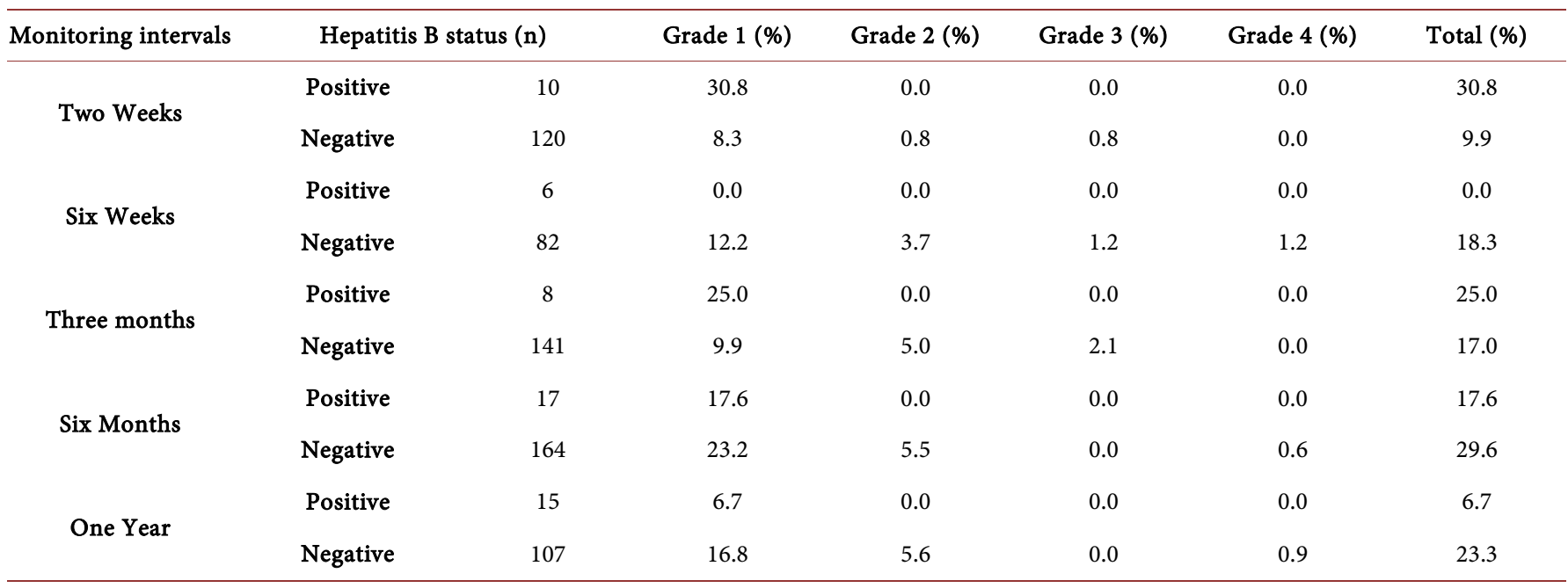

P value-0.01.

line levels as shown in Figure 1. Although many other factors such as viral hepatitis, kidney failure and alcoholism may be associated with an increase in ALT levels in HIV positive patients, antiretroviral drugs have an effect on the liver [6]. Elevated ALT was observed throughout the treatment period for the patients on ART.

The highest total toxicity of 26.5\% (35 patients) and $28.2 \%$ (18 patients) on nevirapine and efavirenz respectively were observed six months after initiation of treatment as indicated in Table 1 . The results of the current study differed from those obtained in similar studies done in Tanzania which had a lower prevalence and Ethiopia which had a higher prevalence, $13 \%$ and $32 \%$ respectively [1] [16]. This difference could be due to different risk factors predisposing the patients to toxicity such as the difference in treatment regimens, CD4 count on commencement of treatment, viral hepatitis co infection, alcohol abuse and pre-treatment ALT elevations and duration of treatment.

Severe hepatotoxicity occurred throughout the course of NNRTI-based therapy (efavirenz and nevirapine). Severe hepatotoxicity (grades 3 and 4) was higher (1.6\%) in patients who were enrolled on efavirenz based regimes than patients who were enrolled on nevirapine based regimes $(1.1 \%)$ as shown in Table 2 . These results were different when compared to those found in a study done in the USA which concluded that severe hepatotoxicity was more common among patients prescribed with nevirapine (15.6\%) than those prescribed with efavirenz $(8.0 \%)$ based regimens [12]. Similarly, other studies conducted in Botswana and Nigeria found that $3.4 \%$ and $16 \%$ of nevirapine and $0.9 \%$ and $8.0 \%$ of efavirenz treated patients developed hepatotoxicity respectively [8] [17].

Most studies have found grade 3 and grade 4 toxicity levels to be between $5 \%-10 \%$ in HIV positive patients treated with combination therapy. However, our study showed higher prevalence of mild toxicity $(16.2 \%$ and $17.1 \%)$ and very low prevalence of severe toxicity $(1.1 \%$ and $1.6 \%)$ irrespective of the type of treatment regimen used as shown in Table 2. The results of the current study were similar to the results of a study done in 


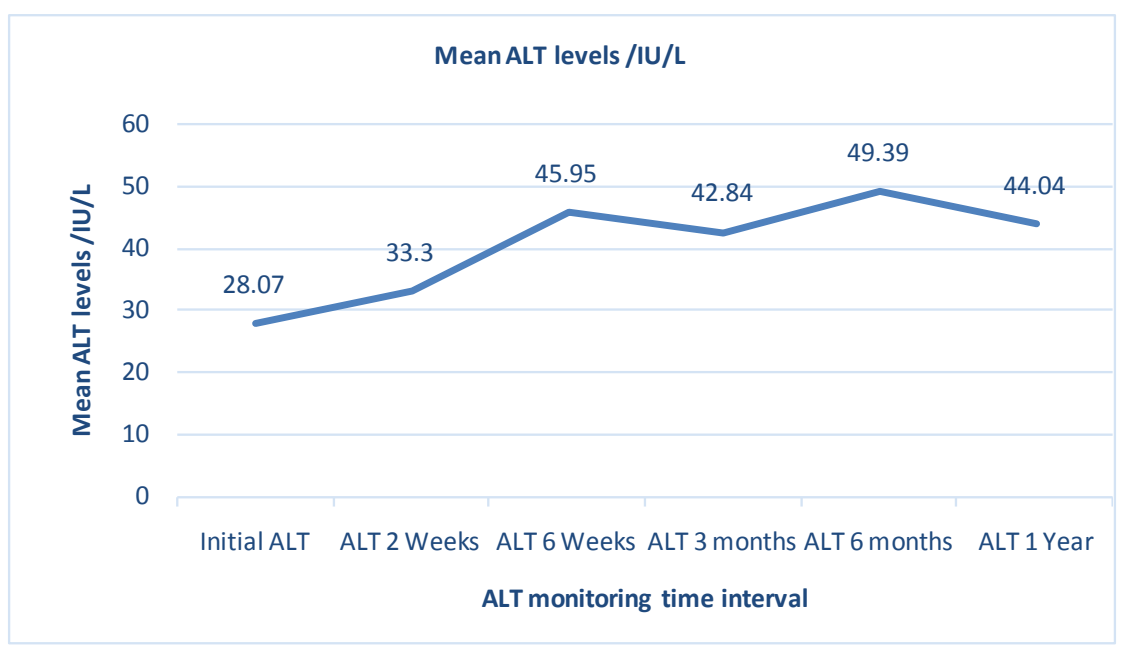

Figure 1. Mean ALT levels at different monitoring intervals. P value-0.082.

Ethiopia which showed higher mild toxicity levels (22.3\% grade 1 toxicity levels, $7.8 \%$ grade 2 toxicity levels) and lower severe toxicity levels ( $1.1 \%$ and $0.74 \%$ for grade 3 and 4 toxicity respectively) [16].

Moreover, a study done in New York City, USA had similar results to our findings with mild toxicity (grade 1 and 2) of $16.7 \%$ and severe toxicity (grade 3 and 4 ) of $1.4 \%$ [10]. Lower results were also found in a study conducted in Tanzania which found $0.3 \%$ severe hepatotoxicity [1] while a higher prevalence of $7 \%$ was found in a study done in Cameroon in mono-infected patients [18]. This difference from the Cameroon study could be due to different treatment regimens and different study designs. The study in Cameroon used nevirapine based regimens only and considered both hepatitis B and C co infection.

As shown in Table 3, patients with viral hepatitis B co infection had highest toxicity at two weeks (30.7\%) and three months (25\%) after initiation of treatment compared to those without viral hepatitis. Thereafter, toxicity was lower in those with hepatitis B viral in comparison to those without hepatitis B virus. However these toxicities were mild (grade 1 toxicity levels). The high toxicity observed could be as a result of the effect of viral hepatitis on the liver causing an increase in ALT.

Hepatitis B co infection has been implicated as a contributing factor to liver injury in patients on ART in similar studies done in South Africa and Switzerland [6] [13].

\section{Conclusion}

The current study found that hepatotoxicity (elevated ALT) levels were experienced throughout the monitoring period, with six months having the highest elevations. Total hepatotoxicity was found to be high (18.0\%). Higher toxicity was observed in patients who were enrolled on efavirenz based treatment regimens compared to those on nevirapine based treatment regimens. Mild toxicity observed in patients who started treatment with viral hepatitis co-infection was high in the first three months, however, no severe toxicity was observed in these patients. 


\section{Authorship}

LPS conceptualized the research and analysed the data; BEvdC supervised the research; MM prepared the manuscript; SRM reviewed the manuscript. All the authors read and approved the manuscript.

\section{Acknowledgements}

The authors would like to acknowledge Windhoek Central and Katutura State Hospitals and MoHSS for approving this research and availing the data used for the study.

\section{References}

[1] Nagu, T.J., Kanyangarara, M., Hwakins, C., Hertmark, E., Chalamila, G., Spiegelman, D., Mugusi, F. and Fawzi, W. (2012) Elevated Alanine Aminotransferase in Antiretroviral-Naive HIV-Infected African Patients. HIV Medicine, 13, 541-548.

[2] Hong, S.Y., Jonas, A., Dumeni, E., Badi, A., Pereko, D., Blomd, A. and Jordan, M.R. (2011) Population-Based Monitoring of HIV Drug Resistance in Namibia with Early Warning Indicators. Journal of Acquired Immune Deficiency Syndromes, 55, 27-31. http://dx.doi.org/10.1097/QAI.0b013e3181f5376d

[3] Aulagnier, M., Janssens, W., De Beer, I., van Rooy, G., Gaeb, E., Hesp, C. and Rinke de Wit, T.F. (2011) Incidence of HIV in Windhoek, Namibia: Demographic and Socio-Economic Associations. PLOS ONE, 6, e25860. http://dx.doi.org/10.1371/journal.pone.0025860

[4] (2015) Global HIV/AIDS: Namibia. http://www.cdc.gov/globalaids/global-hiv-aids-at-cdc/countries/namibia/default.html

[5] Price, J.C. and Thio, C.L. (2010) Liver Disease in the HIV-Infected Individual. Clinical Gastroenterology and Hepatology, 8, 1002-1012.

http://dx.doi.org/10.1016/j.cgh.2010.08.024

[6] Crane, M., Iser, D. and Lewen, S.R. (2012) Human Immunodeficiency Virus Infection and the Liver. World Journal of Hepatology, 4, 91-98.

[7] http://www.cdc.gov/globalaids/global-hiv-aids-at-cdc/countries/namibia/default.html

[8] Eluwa, G.I., Badru, T. and Akpolgbe, K.J. (2012) Adverse Drug Reactions to Antiretroviral Therapy (ARVs): Incidence, Type and Risk Factors in Nigeria. BMC Clinical Pharmacology, 12, 14. http://dx.doi.org/10.1186\%2F1472-6904-12-14

[9] Lemoinea, M. and Ingiliz, P. (2012) Liver Injury in HIV Monoinfected Patients: Should We Turn a Blind Eye to It? Clinics and Research in Hepatology and Gastroenterology, 36, 441447. http://dx.doi.org/10.1016/j.clinre.2012.06.002

[10] Palmon, R., Koo, B.C., Shoultz, D.A. and Dieterich, D.T. (2002) Lack of Hepatotoxicity Associated with Nonnucleoside Reverse Transcriptase Inhibitors. Journal of Acquired Immune Deficiency Syndromes, 29, 340-345. http://dx.doi.org/10.1097/00126334-200204010-00003

[11] Sulkowski, M.S., Thomas, D.L., Mehta, S.H., Chaisson, R.E. and Moore, R.D. (2002) Hepatotoxicity Associated with Nevirapine or Efavirenz-Containing Antiretroviral Therapy: Role of Hepatitis C and B Infections. Hepatology, 35, 182-189. http://dx.doi.org/10.1053/jhep.2002.30319

[12] Dieterich, D.T., Robinson, P.A., Love, J. and Stern, J.O. (2002) Drug Induced Liver Injury Associated with the Use of Non-Nucleoside Reverse Transcriptase Inhibitors. Clinical Infectious Diseases, 38, s80-s89. http://dx.doi.org/10.1086/381450 
[13] Garcia-Jardon, M., Bhat, V.G., Blanco-Blanco, E. and Stepian, A. (2010) Postmortem Findings in HIV/AIDS Patients in a Tertiary Care Hospital in Rural South Africa. Tropical Doctor, 40, 81-84. http://dx.doi.org/10.1258/td.2010.090465

[14] Ministry of Health and Social Services (2014) Directorate of Special Programmes: National Guidelines for Antiretroviral Therapy.4th Edition, Namibia. hivaids@nacop.net

[15] French National Agency for Research on AIDS and Viral Hepatitis (ANRS) (2008) ANRS Scale to Grade the Severity of Adverse Events in Adults. Version 1.0. http://www.anrs.fr/content/download/2242/12805/file/ANRS-GradeEI-V1-En-2008.pdf

[16] Mulu, W., Gidey, B., Chernet, A., Alem, G. and Abera, B. (2013) Hepatotoxicity and Associated Risk Factors in HIV Infected Patients Receiving Antiretroviral Therapy at TelegeHiwot Referral Hospital Bahirder, Ethiopia. Ethiopian Journal of Health Sciences, 23, 217 226.

[17] Wester, C.W., Bussmann, H., Koethe, J., Moffat, C., Vermund, S., Essex, M. and Marlink, R.G. (2009) Adult Combination Antiretroviral Therapy in Sub-Saharan Africa. Lessons from Botswana and Future Challenges, 3, 501-526.

[18] Mbougua, J.B.T., Laurent, C., Kouanfack, C., Bourgeois, A., Ciaffi, L., Calmy, A. and Delaporte, E. (2010) Hepatotoxicity and Effectiveness of a Nevirapine Based Antiretroviral Therapy in HIV-Infected Patients with or without Viral Hepatitis B or C Infection in Cameroon. BMC Public Health, 10, 105. http://dx.doi.org/10.1186/1471-2458-10-105

Submit or recommend next manuscript to SCIRP and we will provide best service for you:

Accepting pre-submission inquiries through Email, Facebook, LinkedIn, Twitter, etc. A wide selection of journals (inclusive of 9 subjects, more than 200 journals)

Providing 24-hour high-quality service

User-friendly online submission system

Fair and swift peer-review system

Efficient typesetting and proofreading procedure

Display of the result of downloads and visits, as well as the number of cited articles Maximum dissemination of your research work

Submit your manuscript at: http://papersubmission.scirp.org/

Or contact wja@scirp.org 